\title{
Proceso didáctico y destrezas en la lectura en niños de primer año de educación básica
}

\author{
Didactic process and reading skills in children in the first year of basic education \\ Processo didático e habilidades de leitura em crianças do primeiro ano do ensino \\ fundamental
}

\section{ARTÍCULO DE REVISIÓN}

Elena del Rocío Rosero Morales ${ }^{1}$
elenadroserom@uta.edu.ec

ORCID: 0000-0001-7004-3207

Myrian Biviana Pérez Constante ${ }^{1}$

mb.perez@uta.edu.ec

ORCID: 0000-0002-7923-9211

\author{
Mercedes Isabel Ruiz Morales ${ }^{2}$ \\ isabelruiz@gmail.com \\ ORCID: 0000-0002-9784-5848
}

\author{
Lilia Cristina Mayorga Jácome ${ }^{3}$ \\ lilia.c.mayorga.j@pucesa.edu.ec \\ ORCID: 0000-0002-0133-1640
}

\author{
1Universidad Técnica de Ambato, Ecuador \\ ${ }^{2}$ Escuela Superior Politécnica de Chimborazo, Ecuador \\ ${ }^{3}$ Pontificia Universidad Católica Sede Ambato, Ecuador
}

Recibido 03 de diciembre 2020 | Arbitrado y aceptado 18 de diciembre 2020 | Publicado en diciembre 2020

\section{RESUMEN}

Se muestra un estudio que describe los diferentes procesos didácticos en el desarrollo de destrezas de lectura en niños y niñas del Primer año de E.G. Básica, con el propósito de mejorar la calidad de enseñanza de las destrezas de la lectura, mediante la aplicación de un proceso didáctico compuesto por el método silábico. El objetivo principal fue analizar los procesos didácticos en las destrezas lectoras en infantes. La metodología empleada para el análisis de la información fue a través del sistema de meta análisis donde lo autores emiten opinión, además, la búsqueda se realizó mediante sistemas acreditados y bases de datos cualificadas certificadas acerca de la variable de las destrezas de la lectura. Como resultados se concluye al Método Silábico como el principal método de enseñanza y aprendizaje significativo del desarrollo de las destrezas de lectura, la metodología de investigación descriptiva ayuda al análisis de los procesos necesarios para adquirir habilidades lectoras y su influencia docente.

Palabras clave: Proceso didáctico; lectura; destrezas; educación básica

\section{ABSTRACT}

A study is shown that describes the different didactic processes in the development of reading skills in boys and girls of the First year of E.G. Basic, with the purpose of improving the teaching quality of reading skills, through the application of a didactic process composed of the syllabic method. The main objective was to analyze the didactic processes in the reading skills in infants. The methodology used for the analysis of the information was through the meta-analysis system where the authors express an opinion, in addition, the search was carried out through accredited systems and certified qualified databases about the variable of reading skills. As results, the Syllabic Method is concluded as the main method of teaching and significant learning of the development of reading skills, the descriptive research methodology helps the analysis of the processes necessary to acquire reading skills and its teaching influence.

Key words: Didactic process; reading; skills; basic education 


\section{RESUMO}

É apresentado um estudo que descreve os diferentes processos didáticos no desenvolvimento da habilidade de leitura em meninos e meninas do primeiro ano do E.G. Básico, com o objetivo de melhorar a qualidade do ensino das habilidades de leitura, através da aplicação de um processo didático composto pelo método silábico. 0 objetivo principal foi analisar os processos didáticos nas habilidades de leitura em bebês. A metodologia utilizada para a análise das informações foi por meio do sistema de metanálise onde os autores expressam uma opinião, além disso, a busca foi realizada em sistemas credenciados e em bases de dados qualificadas e certificadas sobre a variável habilidade de leitura. Como resultados, conclui-se que o Método Silábico é o principal método de ensino e aprendizagem significativa do desenvolvimento da leitura, a metodologia da pesquisa descritiva auxilia na análise dos processos necessários à aquisição da leitura e sua influência no ensino.

Palavras-chave: Processo didático; leitura; habilidades; educação básica

\section{INTRODUCCIÓN}

El proceso didáctico mediante las destrezas de lectura es representado como uno de los principales retos que desde las escuelas de educación prescolar y básica se debe trabajar en pro de la mejora y el desarrollo de didácticas que permitan aglomerar grandes teorías en los procesos de enseñanza y aprendizaje y de esta manera mejorar la calidad educativa del niño. $\mathrm{Al}$ revisar investigaciones relacionadas al tema se encontró diversas fuentes de información necesarias y primordiales en la educación preescolar y primaria de Ecuador.

Es importante recalcar la relevancia de la lectura crítica ayudada por varios textos, fabulas o a su vez cuentos que aportan significativamente a la Educación Básica teniendo a la lectura como punto de partida para desarrollar y aplicar lo aprendido durante sus etapas escolares o en su vida cotidiana, Frías (2014), expone que "La lectura crítica y su incidencia en la destreza de disfrutar de la lectura de las fábulas en los niños/as de cuarto año". (s/p). Por otro lado, en el proyecto Aguilar (2013) expone que "la lectura comprensiva y su incidencia en el aprendizaje significativo de los niños del Cuarto Año de Educación General Básica", indcando que gracias a las diferentes estrategias de comprensión lectora que se han sido aplicada por los docentes, los estudiantes se interesaron en nuevas formas de aprendizaje que los desafíen en su creatividad y en su desarrollo y destrezas, con ello pueden conseguir un medio de actividades diseñadas para la buena comprensión de la lectura y la habilidad de manera satisfactoria en el entorno en que se desenvuelva.

Seguidamente, Molina (2013) en su proyecto "Los cuentos infantiles y su influencia en el hábito lector en los niños de preescolar", menciona que los cuentos infantiles desarrollan diferentes habilidades como la imaginación y creatividad, además de incrementar la comprensión lectora, concentración y atención, esto a la fluidez y facilidad de palabra al comunicarse con las personas de su entorno social.

En el estudio acerca de un "Material Didáctico para la iniciación a la Lectura" desarrollado por Pérez (2016), refiere que el material didáctico es fundamental para enseñar a leer pues no solo se lee en vos alta, sino también en voz silenciosa la cual prepara a los estudiantes para que lean solos y pueden comprender los textos de libro simplemente leyendo con la vista. Además, de fomentar el gusto por la lectura de cuentos solo con imágenes y otros con textos.

Ortiz (2013) manifiesta que es importante que existan instrumentos de evaluación adecuados a las necesidades de los estudiantes de los diferentes niveles de educación, dedicados

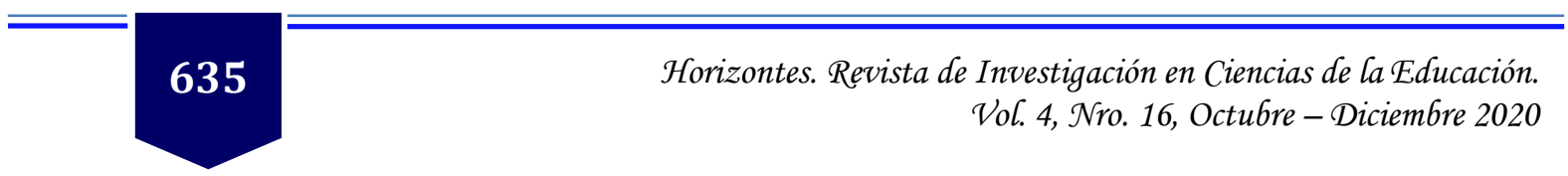


exclusivamente a la lectura comprensiva para que puedan comprender mejor los textos.

La layes en Ecuador ampara a la educación donde mencionando que todas las instituciones educativas toman como base los fundamentos legales y curriculares expuestos por el Ministerio de Educación, los cuales constituyen las normas y reglas educativas del Ecuador, por lo tanto, se toman aspectos fundamentales para la enseñanza aprendizaje como:

Según el Art. 209, Informes de aprendizaje las instituciones educativas deben emitir en un formato oficial definido por el Nivel Central de la Autoridad Educativa Nacional informes parciales, quimestrales y anuales de aprendizaje, que expresen cualitativa y cuantitativamente el alcance de los aprendizajes logrados por el estudiante en cada una de las asignaturas, y en los que se deben incluir recomendaciones para promover el aprendizaje del estudiante (MINISTERIO DE EDUCACIÓN, 2017, pág. 58).

El ministerio de Educación ha creado diversos artículos que favorecen la educación en todos los ámbitos. La educación preescolar es la base de la educación por lo tanto se han impuesto normas las cuales manifiestan que al terminar un periodo de educación se debe presentar evidencia de las actividades realizadas, por los tanto los docentes deben mostrar notas o trabajos hechos por los alumnos, con la intención de verificar el trabajo diario de los mismos.

Pedagógicamente la enseñanza conceptual ha tratado de esquematizar el desarrollo del conocimiento de cada uno de los niños y niñas con sus operaciones intelectuales, entre mayor es el avance de los niños los niveles de pensamiento se vuelven más complejos, y abstractos por lo tanto el aprendizaje será más significativo y eficaz (Pantoja, 2015) y epistemológica se basa específicamente en que la experiencia es la fuente principal de conocimientos, ya que el niño al concebir estas experiencias como aprendizajes significativos para realizar alguna actividad o acción. El niño aprende solo lo que le resulta relevante para él. La interacción de lo que se quiere aprender y la persona que aprenderá son fundamentales para que exista un aprendizaje significativo (Gallego, 2012).

Esta corriente se basa en la construcción de conocimiento que cada uno realiza por eso es fundamental que todos los niños/as tengan un proceso adecuado de Lectura. Cada uno de los procesos que el niño realizara al obtener algún conocimiento debe ser un proceso adecuado e importante para fortalecer sus conocimientos. Además, los sentidos son un parte importante para obtener un aprendizaje (Cruz, 2014).

La problemática presente en la actualidad trata que los docentes en la educación inicial no poseen un proceso didáctico apropiados para el desarrollo de las destrezas lectoras, considerando que en este nivel los infantes se encuentran iniciando el proceso lector, la didáctica ha tenido diversos cambios durante su aplicación, debido que de que los estudiantes logren un aprendizaje adecuado e importante a lo largo de su vida estudiantil, siendo importante para la enseñanza aprendizaje donde se constituye como una disciplina mucho antes que la pedagogía. Las primeras particularidades surgieron por la necesidad de los estudiantes, intentando satisfacer las necesidades de los mismos, esto se pudo lograr gracias a las etapas del estudiante y su desarrollo en las actividades que se realizaban, individualmente o en grupo (Enríquez, 2018). Se debe que tener en cuenta que durante los procesos didácticos al enseñar o aprender cualquier tema, es diferentes en todos los niveles de educación, la edad cronológica y edad mental son factores fundamentales, que las y los docentes 
debe tener en cuenta durante este proceso, si no lo hacen los estudiantes tendrán grandes dificultades en el aprendizaje $y$ no se desarrollan de igual manera.

Además, se deberá considerar la edad de los infantes, su maduración en el aprendizaje $\mathrm{y}$ las necesidades que cada uno de ellos presenta en el momento de aprender cosas nuevas, por ejemplo, no se puede pedir a un niño que no sabe reconocer las letras leer, pues no lo hará y esto solo fomentara la frustración del mismo, por lo tanto, es recomendable que este proceso inicie desde sus primeros años de vida.

El objetivo del presente artículo de revisión bibliográfica es analizar los procesos didácticos en las destrezas lectoras en infantes y como resultados se concluye al Método Silábico como el principal método de enseñanza - aprendizaje significativo del desarrollo de las destrezas de lectura, la metodología de investigación descriptiva ayuda al análisis de los procesos necesarios para adquirir habilidades lectoras y su influencia docente en la utilización de procedimientos didácticos (características de los alumnos, circunstancias ambientales) de los estudiantes, teniendo en cuenta que se debe satisfacer las necesidades internas $\mathrm{y}$ externas del mismo, propiciando un adecuado control y ejecución de las actividades y decisiones que debe tomar el estudiante para influir en la activación de los procesos psicológicos, cognitivos y físicos, con la ayuda del cuerpo docente en todas estas áreas (Guerra, y Montoya, 2015).

\section{MÉTODO}

Se trata de investigación documental donde se resaltó la importancia de revisiones teóricas de autores especialista en destreza de la lectura. Se Hace énfasis a las variable, como didáctica pedagógicas $\mathrm{y}$ aspectos a considera para valorar su calidad. Se aborda el meta-análisis de forma subjetiva realizando una descripción de las opiniones de los autores. Los criterios considerados para extraer las teorías han sido artículos publicados en bases de datos científicas, revistas científicas indexadas, Scielo, Redalyc, Google Académico, Scopus, dando confianza en la información mostrada a lo largo del desarrollo que se presenta del manuscrito.

\section{DESARROLLO Y DISCUSIÓN}

El proceso didáctico se define como una serie de acciones integradas que debe de seguirse ordenadamente por el docente dentro del proceso educativo para el logro de un aprendizaje efectivo. El éxito del proceso didáctico depende del conocimiento, capacidad y actuación del docente para realizarlo con diferentes actividades congruentes y tendientes a la consecución del mismo fin que es facilitar los aprendizajes de los alumnos, porque dichas actividades que son realizadas por el docente están inevitablemente unidas a los procesos de aprendizaje que, siguiendo sus indicaciones, realizan los alumnos.

En didáctica, proceso se refiere al conjunto de actividades ordenadas, interrelacionadas e interdependientes entre sí, tendientes a la consecución de un fin que es el aprendizaje efectivo, cuyas acciones son manejadas $y$ dirigidas por el docente dentro de un periodo de tiempo determinado ya sea dentro o fuera del aula. El proceso didáctico debe ser sumamente planificado, haciendo uso de una forma óptima de los recursos, materiales, financieros y tecnológicos puesto a disposición para el aprendizaje del educando.

En didáctica, proceso se refiere al conjunto de actividades ordenadas, interrelacionadas e interdependientes entre sí, tendientes a la consecución de un fin que es el aprendizaje efectivo, cuyas acciones son manejadas $y$

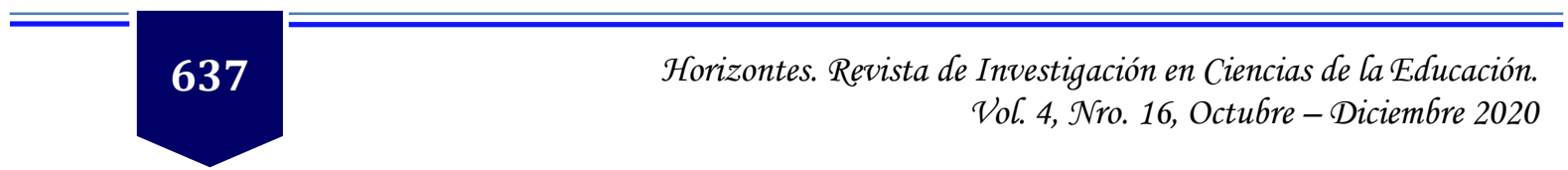


dirigidas por el docente dentro de un periodo de tiempo determinado ya sea dentro o fuera del aula. El proceso didáctico debe ser sumamente planificado, haciendo uso de una forma óptima de los recursos, materiales, financieros y tecnológicos puesto a disposición para el aprendizaje del educando (REICE, 2010). En la antigüedad la educación se implementaba de diversas maneras, por eso cuando la didáctica apareció fue degradada e ignoraba, pues no daba buenos resultados en los estudiantes, por lo tanto, se tuvieron que implementar nuevas estrategias para durante este proceso de enseñanza, mejorado el iteres de los estudiantes en actividades individuales o grupales, sin la necesidad de memorizar o frustrarse, son actividades en las que participaran niños y niñas, las cuales son aplicadas con material didáctico llamativo e interesante para cada uno de ellos.

Durante la creación de la didáctica las aportaciones de la mismo no tuvieron resultados contundentes, pues no existía aun un sistema acabado que determine si se satisfacía las necesidades de todos los que se relacionaban con ella, más bien las polémicas, los debates eran las investigaciones cotidianas, por lo cual han sufrido diversas transformaciones y existen nuevas avances importantes en las investigación de la educación (Abreu, 2017).

Los docentes deben ser humanistas al momento de enseñar, pues la humanidad es parte del ser humano, por lo tanto, al momento de impartir un conocimiento se necesita que todos, ya sean docentes o estudiantes, sientan empatía uno por otro teniendo en cuenta las necesidades de cada uno de ellos.

Desde la perspectiva de los docentes la didáctica es humanista, se parte de la practica real de la educación es impórtate que los estudiantes participen en todo este procesos, ya que gracias a esto se puede buscar actividades cotidianas que beneficien al estudiantes en todos los ámbitos educativos, esto además constituye una parte primordial del docente ya que él se debe escuchar así mismo y a los estudiantes para lograr u procesos didáctico humanista (Cruz, 2014).

Sin lugar a dudas el proceso didáctico en la educación es una de las partes más importantes del mismo, por lo tanto, es fundamental aclarar que en la actualidad no se aplica los procesos didácticos solamente en las matemáticas y la lectura como se hacía en la antigüedad, sino por el contrario ahora estos procesos su una aparate fundamental en la educación, esencialmente en la primera infancia (educación inicial).

La didáctica en su concepto inicial fue la representación mental de los objetos del conocimiento, pero luego cambio pues se centró específicamente en el estudio de la educación sobre la enseñanza aprendizaje específicamente en la matemática y el lenguaje, son áreas muy amplias para estudiarlas cada una de ellas, además algunas regiones tomaron mayor interés por la didáctica y dejaron de lado la pedagogía (Leal, 2015)

Aunque en la era moderna los procesos didácticos se han transformado, en la educación inicial ha mantenido su esencia, la cual muestra que los niños aprenden de mejor manera con los recursos materiales del entorno o elaborados según sus necesidades, se toma en cuenta esto pues es fundamental que todos los estudiantes tengan un aprendizaje con materiales elaborados por ellos mismos, esto fomenta la creatividad y acelera en proceso didáctico al aprender las habilidades necesarias para toda su vida.

Al momento de hablar sobre la lectura podemos decir que el proceso didáctico que 
se utiliza son las imágenes y el reconocimiento de las mismas, además de descifrar las letras o palabras que observamos al momento de leer un texto.

Por otro lado durante las investigaciones se llegó a la conclusión que los materiales didácticos son importantes para que se desarrolló un proceso adecuado, por lo tanto, el material didáctico en un alternativa muy llamativa para el aprendizaje en los estudiantes, pues los mismos prefieren un aprendizaje practico - lúdico con elementos reales que activen su gusto por aprender y que estimulen su memoria, motricidad fina y gruesa, entre otros. (Gallego, 2012).

A continuación, se describe el proceso de los métodos investigados:

\section{Método Alfabético o Deletreo}

En la antigüedad se inició la enseñanza de la lectura como una forma sistemática, en la cual se aplicaba el Método alfabético. El mismo se utiliza hace muchos años atrás (Edad antigua, media y moderna) el cual recibió este nombre por el proceso que se utiliza pues su base es el alfabeto.

Inicialmente se comienza con la observación, la cual consiste en mostrar las letras. Lo más recomendable es que se aprendan de 20 a 25 letras al principio, después se unen unas a otras para formar palabras y leerlas despacio.

Esto era muy confuso, las letras eran poco comprendidas, además de que aprendían su nombre con gran dificultad al igual que las oraciones compuestas por 2 o más letras, Según (BOCHE, 2014), su aplicación requiere del seguimiento de estos pasos. Se sigue el orden alfabético para su aprendizaje.

Se empieza pronunciando el nombre de las letras del alfabeto, por ejemplo: a; be, ce; de; e; efe; entre otros. A continuación, se va relacionando la lectura y escritura de las letras, después se aprende las consonantes las cuales se combinan con las vocales y se elaboran silabas (silabas directas, silabas inversas y mixtas), finalmente se aprende la acentuación y puntación, este método se enfatiza principalmente por la lectura mecánica que se aprende (Ortego, López, y María Lourdes Álvarez, 2015).

\section{Método Silábico}

La educación insatisfactoria en la antigüedad busco otro método para satisfacer sus necesidades por lo que se elaboró el método silábico el cual facilitaría la enseñanza de la, el método silábico se les adjudica especialmente a los pedagogos (Ortego, López, y María Lourdes Álvarez, 2015) en el cual el método se define un proceso más corto pero efectivo que consiste especialmente en la enseñanza de las vocales, consonantes las cuales van cambiando entre si y formando silabas y luego palabras.

A continuación, el método silábico:

- Se enseñan las vocales enfatizando en la escritura y la lectura.

- Las consonantes se enseñan respetando su fácil pronunciación, luego se pasa a la formulación de palabras, para que estimule el aprendizaje.

- Cada consonante se combina con las cinco vocales en sílabas directas así: ma, me, mi, mo, mu, etc.

- Cuando ya se cuenta con varias sílabas se forman palabras y luego se construyen oraciones. e) Después se combinan las consonantes con las vocales en sílabas inversas así: am, em, im, om, um y con ellas se forman nuevas palabras y oraciones.

- Después se pasa a las sílabas mixtas, a los diptongos, triptongos y finalmente a las de cuatro letras llamadas complejas 
- Con el silabeo se pasa con facilidad a la lectura mecánica, la expresiva y la comprensiva.

- El libro que mejor representa este método es el silabario.

- Método nuevo:

Se define este como el método global el cual está muy bien recibido por los docentes, lo cuales manifiestan que este método y el de Decroly son las apropiados para enseñar lectura. Para esto se manifiesta que existen tres etapas del aprendizaje las cuales son:

- Percepción global de la frase

- Percepción global de la frase

- Análisis y síntesis de la palabra

Cada una de estas etapas corresponde a una etapa de desarrollo diferente e exclusiva, teniendo en cuenta que esto no puede ser fijado por nadie, los niños y niñas son diversos y diferentes, durante estas etapas las observaciones espontaneas anuncian $\mathrm{y}$ prepara la etapa siguiente, determinando así que se ha logrado cada uno de los objetivos que se ha propuesto, (Cléries, 2016). A continuación, se explica cada una de las etapas:

\section{Primera etapa: la frase}

\section{a) Educación de la memoria visual}

Para esto se inicia con juegos educativos: identificación de colores, formas, lugares, posiciones, etc. Esto constituye una gran parte del proceso de la lectura, son ejercicios de atención y crean habilidades audiovisuales. Se puede identificar que el niño no está apto para la lectura cuando sus dibujos son indescifrables, además de los signos abstractos, como los que componen las palabras y las frases.
Para el método global la memoria visual es más importante que el método fonético, pues al observar diversas cosas el niño mejora su memoria y esto ayuda a que posteriormente no tenga ningún problema al recordad nombres, figuras, sonidos, etc.

\section{b) Estudio de la frase}

El principio del método global consiste en asociar la expresión a la actividad global del alumno, para esto se iniciará con la frase, que resulte de un ejercicio de observación o de una conversación familiar. Durante este proceso de la frase, se incluirán textos cortos los cuales pueden ser alargados dependiendo de las posibilidades de los estudiantes, además de tener en cuenta sus necesidades.

Se inicia este proceso con la ayuda de su entorno, cada una de las cosas que el niño observe puede ser utilizada para un cuento o una historia que posteriormente relacione con lo que quiere hablar o explicar.

$\mathrm{Si}$ el asunto agrada a los estudiantes se pueden incluir frases más elaboradas, teniendo en cuenta el tiempo que dura su atención a una sola actividad sin la necesidad de obligarlos, esto solo fomentará un mal recuerdo de la lectura. Es inapropiado para los docentes manifestar que los estudiantes estudian 40 tiras y se sabe 20 , no se está observando su progreso al determinar las palabras esenciales de una frase, sino solamente su capacidad de retener palabras. (Costa y López, 2015).

\section{Segunda etapa: la palabra}

\section{a) Adquisición del vocabulario}

Gracias al leguaje escrito se puede enriquecer el vocabulario, la primera etapa ha servido como base para que el estudio se la frase sea mantenida, los textos expresaran lo que siente el niño. 


\section{b) El vocabulario}

Es importante señalar que los niños leen por la idea, las palabras que ellos conocen les sirven como guía para comprender y leer lo que continúa en el texto. Mientras más palabras conozcan más fácil será para el leer los textos.

Es verdad que no se necesitan conocer todas las palabras, pero sí que las palabras principales sean reconocidas sin la ayuda del contexto. Además, esta condición es fundamental pala el análisis preparatorio. (Costa y López, 2015).

\section{Tercera etapa: análisis y síntesis de la palabra}

\section{a) Análisis}

Después de que el vocabulario es adquirido, los estudiantes que tiene una mayor comprensión de las palabras empiezan a comparar, pero sin un determinado.

\section{b) Análisis espontaneo}

Para eso se observan las actitudes y acciones del niño, sin la necesidad de implementar alguna evaluación, por lo tanto, el estudio prematuro delas letras solo traerá consecuencias negativas tanto desde el punto del mecánico como de la comprensión lectora. Además de esto, el análisis de la palabra comprende grados, lo cuales son complejos para los estudiantes.

\section{c) El método}

Durante este tiempo las comparaciones entre consonantes son muy escasas, el niño solo toma en cuenta las vocales y silabas.

\section{Silabas: \\ - Monosílabos \\ - Comparar palabras y extraer silabas \\ 2. Vocales}

Durante este proceso los sonidos más largos y fuertes son lo que el oído percibe con más atención $(\mathrm{a}, \mathrm{e}, \mathrm{o})$. En algunos casos los niños y niñas que tienen un conocimiento adecuado de los sonidos aprenden a leer más rápido, mientras que los que no saben reconocerlos les tomas tiempo relacionarlos entre sí. (Cléries, 2016). También se realizó una investigación acerca de los tipos de lectura y como aporta en educación a cada uno de ellos en los niños y niñas, que desarrollan las destrezas de lectura.

\section{Tipos de lectura}

La lectura es una de las actividades más importantes en la vida del ser humano, por lo tanto, esto debe ser muy agradable, podemos manifestar que en la actualidad existe un bajo aporte a la lectura por parte de la juventud, no existen actividades o estrategias que fomenten la lectura como algo bueno. Se puede observar que en la actualidad la mayora de personas piensa que la lectura es una pérdida de tiempo, sim embargo podemos decir que la lectura es una acción que realizamos todos los días, incluso sin quererlo, simplemente al pasar por una tiendo o por un kiosco, se nos preséntala oportunidad de leer, para que la lectura tenga una valor agradable hacia las personas debemos fomentar el mismo desde las primeras etapas del ser humano, no obligándoles a leer, sino simplemente dejando que su imaginación crezca y cree un cuento, una historia, un poema, una canción, aprovechando cada uno de esos actos para decirle a los niños y niñas que todo está muy bien y magnifico, esto constituye un ente hacia una mejor sociedad lleno de personas seguras de sí mismo y sabiendo que todo lo que se propongan lo logren, para esto hay la docente debe conocer cada uno de los tipos de la lectura y saber cuál se debe aplicar en ,los

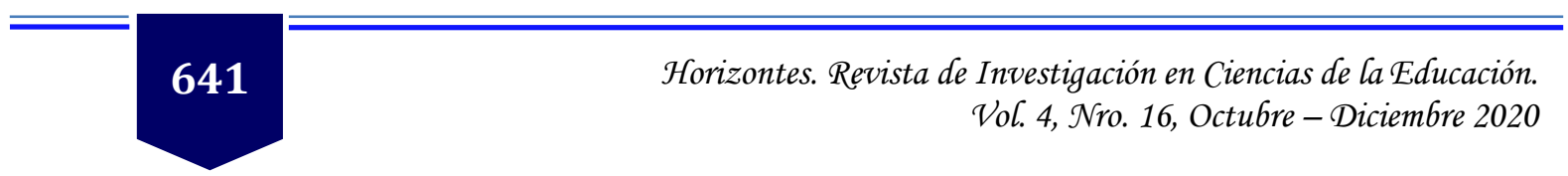


momentos más importantes de sus estudiantes.(Ortego, López, y María Lourdes Álvarez, 2015).

Lectura oral: Esta lectura se realiza cuando una o varias personas leen en voz alta para comprender mejor, algunas personas hacen esto, al escuchar en sonido y las palabras quedan estas grabadas en el cerebro y que cuando escuchen esta palabra otra vez sea más fácil para el reconocerla y recordarla. Además, es muy útil ya que ayuda a compartir experiencias o hechos que una persona ha realizado en un tiempo determinado.

Lectura silenciosa: En este caso la persona simplemente lo lee con la mente, consideran que es más fácil aprender las cosas a través de sus ojos, sin la necesidad de hablar o realizar alguna otra actividad para comprender lo que lee. Las personas que realizan esta lectura son aquellas que crean su propio mundo para leer y comprender según ellos consideren adecuado.

Lectura superficial: En esta lectura simplemente la persona que lee o comprender, solo lo hace para conocer el tema que se está tratando sin la necesidad de leer todo correctamente, esto significa que la persona solo busca comprender la idea general mas no los detalles y características que se están dando en ese momento.

Lectura selectiva: Este tipo de lectura se realiza cuando las personas buscan algo en específico, características, opiniones, etc., simplemente buscara lo que le interese y eliminara todo aquello que para él sea innecesario, también se lo puede describir como una lectura de búsqueda, en este caso no hay la necesidad de leer minuciosamente todo el texto solo se centrara en las palabras claves que le ayudara al encontrar lo que busca.

Lectura comprensiva: En esta lectura la persona que lee busaca comprender todo el texto que lee sin importar si es lo que buscaba o no, esto se practica de manera lenta, para comprender esto hay que comprender todo el contenido y esto lleva tiempo.

Lectura reflexiva y crítica: En esta lectura el lector solo analizara el texto que tenga en frente y así lo aceptara como verdad absoluta, pues él considera que si leyó esto está bien. Un lector critico por otra parte es una persona que lee el texto y lo compara con otras afirmaciones o con su propia experiencia, en algunos casos puede no estar de acuerdo con el texto que lee pues al momento de comparar el comprenderá si es verdad o no lo que el autor del libro quiere expresar.

Lectura recreativa: En esta lectura se comenta el placer por la lectura, no importa el tiempo ni la velocidad con la que una persona lea simplemente para el lector esto debe ser una experiencia placentera, además en este tipo de lectura se mantiene activo el cerebro y mejora la creatividad e imaginación al momento de leer (Omar Abreu, 2017).

\section{CONCLUSIÓN}

Se concluye que el proceso didáctico más utilizado por las docentes en Educación Básica es el Método Silábico el cual se desarrolla principalmente con el inicio del aprendizaje de las vocales, posteriormente se aplica la unión de consonantes y vocales, de la misma forma se combinan las consonantes y vocales inversas, consecuentemente los niños y niñas empiezan a desarrollar las silabas mixtas como los diptongos, triptongos y las complejas.

El nivel de desarrollo de destrezas de lectura en los niños y niñas se encuentra en desarrollo al encontrarse dentro del proceso de iniciación de la lecto escritura, pueden reconocer imágenes, responder preguntas coherentes y repetir las palabras con claridad, 
tomando en cuenta que representan un alto nivel de desarrollo cognitivo y a su entorno social.

El proceso didáctico ha llegado a ser de suma importancia en los primeros años de la enseñanza del aprendizaje de la lectura, principalmente teniendo como base la aplicación de material didáctico que aporta de manera significativa a las herramientas educativas compuestas por actividades innovadoras y creativas, durante el proceso de aplicación. Por lo tanto, se concluye que el proceso didáctico es la parte fundamental para la enseñanza aprendizaje.

\section{REFERENCIAS}

Abreu, M. C. (2017). La Didáctica: Epistemología y Definición en la Facultad de Ciencias Administrativas y Económicas de la Universidad Técnica del Norte del Ecuador. Obtenido de La Didáctica: Epistemología y Definición en la Facultad de Ciencias Administrativas y Económicas de la Universidad Técnica del Norte del Ecuador:

https://scielo.conicyt.cl/scielo.php?script =sci_arttextypid $=$ S0718-

50062017000300009

Aguilar, M. G. (2013). Repositorio Universidad Técnica de Ambato. Obtenido de http://repositorio.uta.edu.ec/jspui/handle $/ 123456789 / 7224$

Boche, M. X. (2014). "destrezas requeridas para el aprendizaje de la lecto-escritura que deben ser tomadas en cuenta en la transición de preparatoria a primer grado. (estudio realizado en un grupo de preparatoria del colegio de señoritas: "el Sagrado Corazón" El Naranjo)". Obtenido de "destrezas requeridas para el aprendizaje de la lecto-escritura que deben ser tomadas en cuenta en la transición de preparatoria a primer grado. (estudio realizado en un grupo de preparatoria del colegio de señoritas: "El Sagrado Corazón" El Naranjo)":
http://biblio3.url.edu.gt/Tesario/2014/0 5/24/Contreras-Maria.pdf

Cléries, X. (2016). La Comunicacion.- Una Competencia esencial para los profesionales de la salud. Barcelona: Masson. Obtenido de https://www.masonica.es/libro/el-librodel-aprendiz-mason_51916/

Costa, M., y López, E. (2015). Manual del Educador Social 2. Madrid: editors. Obtenido de https://ocw.unican.es/pluginfile.php/157 5/course/section/2034/tema_06c.pdf

Cruz, E. Y. (2014). La didáctica es humanista. Obtenido de La didáctica es humanista: http://www.scielo.org.mx/scielo.php?scri pt=sci_arttextypid=S018526982014000300014

Frías, D. (2014). Repositorio Universidad Técnica de Ambato. Obtenido de http://repositorio.uta.edu.ec/jspui/handl e/123456789/6975

Gallego, M. M. (e 2012). El material didáctico para la construcción de aprendizajes significativos. Obtenido de el material didáctico para la construcción de aprendizajes significativos: file://C:/Users/HP/Downloads/DialnetElMaterialDidacticoParaLaConstruccionD eAprendizaje-5123813.pdf

Guerra Harriette, D. R., y Montoya Rodríguez, C. (marzo de 2015). Procedimientos didácticos para la solución de problemas en la enseñanzaaprendizaje de la Biología Química. Obtenido de Procedimientos didácticos para la solución de problemas en la enseñanzaaprendizaje de la Biología

Química: https://www.redalyc.org/pdf/4757/4757 47191004.pdf

Enríquez Clavero, J. (2018). ¿Qué didáctica desarrollar, la general y/o las particulares? Reflexiones desde su epistemología. Obtenido de ¿Qué didáctica desarrollar, la general y/o las particulares? Reflexiones desde su epistemología: http://scielo.sld.cu/scielo.php?script=sci_ arttextypid=S2077-28742018000300010

Leal, A. Z. (2015). Pedagogía Y Didáctica: Esbozo De Las Diferencias, Tensiones Y

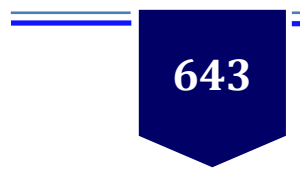


Relaciones De Dos Campos. Obtenido de pedagogía y didáctica: esbozo de las diferencias, tensiones y relaciones de dos campos:

file://C:/Users/HP/Downloads/DialnetPedagogiaYDidactica-5601292.pdf

Ministerio De Educación. (2017). Reglamento general a la ley orgánica de educación intercultural. Obtenido de reglamento general a la ley orgánica de educación intercultural:

https://educacion.gob.ec/wp-

content/uploads/downloads/2017/05/R

eglamento-General-Ley-Organica-

Educacion-Intercultural.pdf

Molina, X. (12 de Junio de 2013). Los cuentos infantiles y su influencia en el hábito lector en los niños de preescolar del centro de educación básica "lemcis" de la ciudad de ambato provincia de tungurahua. Obtenido de

https://repositorio.uta.edu.ec/handle/12 $3456789 / 4160$

Ortego, M. d., López, S., y María Lourdes Álvarez, M. d. (2015). Ciencias psicosociales II. Universidad de la Cantabria: Course Ware. Obtenido de https://ocw.unican.es/pluginfile.php/157 5/course/section/2034/tema_06c.pdf

Ortiz, M. P. (2013). Repositorio Universidad Técnica de Ambato. Obtenido de http://repositorio.uta.edu.ec/jspui/handl e/123456789/7293

Pantoja, V. (2015). FUNDAMENTACIONES DE LA LECTURA. Revista Iberoamericana de Educacion Superior, 6(15), 129-144. Obtenido de https://www.sciencedirect.com/science/ article/pii/S200728721530007X

Pérez, s. (2016). "MATERIAL DIDÁCTICO PARA LA INICIACIÓN A LA LECTURA". Obtenido de

http://recursosbiblio.url.edu.gt/tesiseorti z/2016/05/84/Perez-Skarltt.pdf

Recursos educativos tic de información,colaboracion y aprendizaje. (2019). Revista de medios y educación, 39(2), 69-81

REICE. (2010). Revista Iberoamericana sobre Calidad, Eficacia y Cambio en Educación. Obtenido de Introducción a los procesos de calidad: https://www.redalyc.org/pdf/551/55119 084001.pdf 